6-1998

\title{
Semiclassical Formula for Oscillator Strengths of Atomic Spectra in External Fields
}

\author{
V. Kondratovich \\ William \& Mary \\ John B. Delos \\ William \& Mary, jbdelos@wm.edu
}

Follow this and additional works at: https://scholarworks.wm.edu/aspubs

Part of the Physics Commons

\section{Recommended Citation}

Kondratovich, V. and Delos, John B., Semiclassical Formula for Oscillator Strengths of Atomic Spectra in External Fields (1998). Physical Review A, 57(6), 4654-4661.

https://doi.org/10.1103/PhysRevA.57.4654

This Article is brought to you for free and open access by the Arts and Sciences at W\&M ScholarWorks. It has been accepted for inclusion in Arts \& Sciences Articles by an authorized administrator of W\&M ScholarWorks. For more information, please contact scholarworks@wm.edu. 


\title{
Semiclassical formula for oscillator strengths of atomic spectra in external fields
}

\author{
Vladimir Kondratovich and John B. Delos \\ Department of Physics, College of William and Mary, Williamsburg, Virginia 23187
}

(Received 12 January 1998)

\begin{abstract}
In a previous Rapid Communication [Phys. Rev. A 56, R5 (1997)] we reported a semiclassical formula describing oscillator strengths for transitions to a high Rydberg state of a hydrogen atom in an electric field. The formula relates the oscillator strength for an atomic transition into a Rydberg state to the angular distribution of outgoing electron waves and to the density of classical tori. Here we give a derivation of that formula and we suggest some generalizations. We compare the formula with experimental measurements of the absorption spectrum of hydrogen in an electric field. This absorption spectrum consists of quasidiscrete resonances superposed on a smooth background. We find that this background has a hitherto unexplained structure that was visible but not recognized in the experiment. The background absorption is related to electron orbits that directly escape from the atom, and its structure is related to the angular distribution of outgoing electrons. [S1050-2947(98)10606-6]
\end{abstract}

PACS number(s): 32.60. $+\mathrm{i}, 03.65 . \mathrm{Sq}, 32.80 .-\mathrm{t}$

\section{INTRODUCTION}

We consider the absorption spectrum to highly excited states of a hydrogenic atom in applied electric and/or magnetic fields. The oscillator strength for the transition from a given initial state $\mathbf{i}$ to a final state $\mathbf{n}$ is defined as

$$
f_{\mathbf{n}}^{\mathbf{i}}=\left[2 m_{e}\left(E_{\mathbf{n}}-E_{\mathbf{i}}\right) / \hbar^{2}\right]|\langle\mathbf{i}|D| \mathbf{n}\rangle|^{2},
$$

where $D$ is the relevant component of the dipole operator. In a previous paper [1], we presented a simple semiclassical formula for this oscillator strength, and we showed how the formula could explain patterns that are observable in atomic spectra. That formula was

$$
f_{\mathbf{n}}^{\mathbf{i}}=\left.(8 \pi)\left(\frac{m_{e}^{2} a_{0}^{2}}{\hbar^{2}}\right)\left(E_{\mathbf{n}}-E_{\mathbf{i}}\right)\left|\frac{\partial(E, \beta)}{\partial\left(I_{1}, I_{2}\right)}\right| \mathcal{Y}\left(\theta_{\mathbf{n}}\right)\right|^{2} .
$$

The formula combines quantum, semiclassical, and classical concepts. $\mathcal{Y}(\theta)$ is the angular distribution of electron waves going out from the atom as a result of excitation by light. For example, if the initial state $\mathbf{i}$ is spherically symmetric and the light is linearly polarized along the $z$ axis, $\mathcal{Y}(\theta)$ is a $p_{z}$ wave, proportional to $\cos \theta$. This quantity arises from quantum theory.

$I_{1}$ and $I_{2}$ are classical actions. The Hamilton-Jacobi equation for the Stark system is separable in semiparabolic coordinates, $\beta$ is the separation constant, and there exists a canonical transformation from the conserved quantities $(E, \beta)$ to the action variables $\left(I_{1}, I_{2}\right)$. The Jacobian of that transformation appears in Eq. (1.2). Semiclassical theory tells us that each quantum state $\mathbf{n}$ corresponds to an "eigentrajectory", having quantized values of these action variables.

Finally, analysis of the classical orbits of the Stark system shows that for each trajectory there is a unique "angle of ejection from the atom" $\theta_{I_{1}, I_{2}} \equiv \theta_{\mathbf{n}}$ : a classical electron moving radially outward from the nucleus at this angle will find itself on the torus having action variables $\left(I_{1}, I_{2}\right)$ (which then corresponds to the quantum state $\mathbf{n}$ ).

In this paper we present the proof of this formula. Actually we establish a more general formula, Eq. (4.7), which is not directly tied to the Stark system, but which applies more generally to one-electron atoms in fields, if the spectrum is regular.

In our previous paper, we tested the formula by comparison with numerical quantum calculations. Here we test it by comparison with experiments carried out by Rottke and Welge [2]. This comparison forces us to examine another issue. In the Stark system, the absorption spectrum consists of narrow lines superposed on a smooth continuum. Is there a formula analogous to Eq. (1.2) that describes the continuum? In this paper we derive and test a new semiclassical formula for that continuous absorption. Intensity for absorption into a continuum is described by an oscillator-strength density, $D f(E)$; we will show that in this case $D f(E)$ is given by

$D f(E, F)=(8 \pi)\left(m_{e}^{2} a_{0}^{2} / \hbar^{4}\right)\left(E-E_{\mathbf{i}}\right) \int_{\theta_{c}(E, F)}^{\pi}|\mathcal{Y}(\theta)|^{2} \sin \theta d \theta$

where $\theta_{c}$ is a critical ejection angle dividing bound from escaping orbits.

Our derivation is based on the following assumptions. (a) The final state $\mathbf{n}$ is high lying, so it can be described by a semiclassical approximation. (b) The initial state $\mathbf{i}$ is localized in comparison with the final state. (c) The external fields are weak enough to allow the Coulomb field to dominate in the region of localization of the initial state. (d) The system (electron in combination of external fields and Coulomb field) has cylindrical symmetry and a regular spectrum (some of our formulas presume that $m=L_{z} / \hbar=0$, but this is not essential). (e) Near the nucleus the electron trajectories in the final state are similar to those for zero-energy Coulomb scattering. (f) The system satisfies a certain "nondegeneracy condition," which will be defined later.

We derive the formula (1.2) for discrete states in the next two sections, and then convert it to the formula (1.3) for continuum absorption in Sec. V. Section VI contains our comparisons with measurements. Further generalizations of the formulas are sketched in Appendix B. 


\section{SEMICLASSICAL WAVE FUNCTION FOR THE FINAL STATE}

Let us consider a regular system having cylindrical symmetry around the $z$ axis. The orbital angular momentum $L_{z}$ around this axis is conserved, the azimuthal angle is ignorable, and the reduced two-dimensional motion may be described by coordinates $(\rho, z)$ or $(r, \theta)$ or $(u, v)$ $=\left(r^{1 / 2} \cos \theta / 2, r^{1 / 2} \sin \theta / 2\right)$. We said above that we assume that "the system has a regular spectrum." More precisely, we assume that there is a region of phase space in which the trajectories are regular, they foliate that region as a continuous family of tori, and each torus is labeled by two action variables,

$$
I_{j}=\frac{1}{2 \pi} \oint_{C_{j}} \mathbf{p} d \mathbf{q}
$$

where $C_{j}$ are the irreducible loops on the torus.

We allow the possibility that other parts of phase space may contain chaotic trajectories, and also we allow the possibility that small zones of chaotic behavior may be present even in the region of interest. In the region of interest, the trajectories must be sufficiently regular to allow the standard techniques of Einstein-Brillouin-Keller-Marcus quantization to be used.

This implies that besides the energy $E$, there is a second (exact or approximate) conserved quantity, which we name $\beta$; tori are equivalently labeled by $(E, \beta)$ or $\left(I_{1}, I_{2}\right)$; $\partial(E, \beta) / \partial\left(I_{1}, I_{2}\right) \neq 0, \infty$. No further specification is yet given of this quantity $\beta$ (except that we take it to be dimensionless).

In the regular region of phase space there is a canonical transformation from the original Cartesian variables $(\mathbf{p}, \mathbf{q})$ to action-angle variables $(\mathbf{I}, \boldsymbol{\phi})$, and that transformation admits (type-2) generating functions

$$
S(\mathbf{q}, \mathbf{I})=\int^{\mathbf{q}} \mathbf{p}\left(\mathbf{q}^{\prime}, \mathbf{I}\right) d \mathbf{q}^{\prime} .
$$

If the regular region of phase space is sufficiently large, then a regular family of quantum states builds itself upon the "eigentori" - those tori for which the two action variables are quantized as

$$
I_{j}=\left(n_{j}+\nu_{j} / 2\right) \hbar, \quad j=1,2
$$

(most commonly $\nu_{j}=1$ ). Miller [3] and Littlejohn [4] gave a semiclassical formula for the corresponding eigenfunction,

$$
\begin{aligned}
\Psi_{\mathbf{I}}\left(\mathbf{q}^{\prime}\right)= & (2 \pi)^{-k / 2} \sum_{j}\left|\left(\partial^{2} S_{j}\left(\mathbf{q}^{\prime}, \mathbf{I}\right) / \partial \mathbf{q}^{\prime} \partial \mathbf{I}\right)\right|^{1 / 2} \\
& \times \exp \left\{i\left[S_{j}\left(\mathbf{q}^{\prime}, \mathbf{I}\right) / \hbar-\mu_{j} \pi / 2\right]\right\} .
\end{aligned}
$$

The sum is over the various "branches" of the torus that project to the point $\mathbf{q}^{\prime}, k$ is the number of variables in the set $\mathbf{q}$, and $\left|\partial^{2} S\left(\mathbf{q}^{\prime}, \mathbf{I}\right) / \partial \mathbf{q}^{\prime} \partial \mathbf{I}\right|$ means the absolute value of the determinant. (Miller and Littlejohn include a factor $(i \hbar)^{-k / 2}$, which we deliberately omit.) This wave function (as we define it) is normalized such that [Appendix A]

$$
\int\left|\Psi_{\mathbf{I}}\left(\mathbf{q}^{\prime}\right)\right|^{2} d \mathbf{q}^{\prime}=1 .
$$

The above equation holds in Cartesian coordinates $\mathbf{q}^{\prime}$ $=(x, y, z)$.

We want the semiclassical form in the reduced twodimensional space $\mathbf{q}=(r, \theta)$ or $(\rho, z)$ or $(u, v)$. The Cartesian generator and the prefactor are reexpressed in the desired coordinates by, for example,

$$
\begin{gathered}
S\left(x, y, z, I_{1}, I_{2}, L_{z}\right)=S\left(r, \theta, I_{1}, I_{2}\right)+L_{z} \phi, \\
\left|\frac{\partial^{2} S}{\partial(x, y, z) \partial\left(I_{1}, I_{2}, L_{z}\right)}\right|=\left|J_{r \theta}\right|\left|\frac{\partial^{2} S}{\partial(r, \theta) \partial\left(I_{1}, I_{2}\right)}\right|,
\end{gathered}
$$

where

$$
J_{r \theta}=\left|\frac{\partial(r, \theta, \phi)}{\partial(x, y, z)}\right|=\left(r^{2} \sin \theta\right)^{-1} .
$$

Analogous expressions hold for $(u, v)$ or $(\rho, z)$ coordinates.

Our primary assumption is that the semiclassical form such as the one in $(r, \theta, \phi)$,

$$
\begin{aligned}
\Psi_{\mathbf{I}}(r, \theta, \phi)= & (2 \pi)^{-3 / 2} \sum_{j} J_{r \theta}^{1 / 2}\left|\frac{\partial^{2} S_{j}\left(r, \theta, I_{1}, I_{2}\right)}{\partial(r, \theta) \partial\left(I_{1}, I_{2}\right)}\right|^{1 / 2} \\
& \times \exp \left\{i\left[S_{j}\left(r, \theta, I_{1}, I_{2}\right) / \hbar+L_{z} \phi / \hbar-\mu_{j} \pi / 2\right]\right\}
\end{aligned}
$$

[or the corresponding form in $(\rho, z, \phi)$ or $(u, v, \phi)]$, describes the excited state over most of the two-dimensional space, and that this wave function is normalized to unity in that space.

\section{FINAL-STATE WAVE FUNCTION NEAR THE NUCLEUS}

Equation (2.9) cannot be correct near the nucleus, or indeed anywhere on the $\pm z$ axes. There it diverges as $\left(r^{2} \sin \theta\right)^{-1}$, so a different formula must be used. It is impossible to give a formula that will cover all cases. We propose that most cases will be covered by one simple assumption that can be stated in any of four ways.

(a) Near the nucleus, the trajectories associated with the semiclassical approximation for the excited state are similar to those for cylindrically symmetric zero-energy Coulomb scattering [Fig. 1(a)]. There exists an angle $\theta_{f}$ at which a trajectory comes on a straight line exactly to the nucleus, turns around and goes back out on itself. Nearby the trajectories form parabolas symmetric about that line. We further assume that $\theta_{f}$ varies smoothly as a function of the second conserved quantity, with $\left(\partial \theta_{f} / \partial \beta\right)_{E} \neq 0$. We are saying that $\left(E, \theta_{f}\right)$ provide alternative labels for the tori, $\left|\partial\left(E, \theta_{f}\right) / \partial\left(I_{1}, I_{2}\right)\right| \neq 0, \infty$.

(b) When represented in $( \pm u, \pm v)$ space, the torus has a regular projection onto a domain around the origin $(u=0$, $v=0)$, and in the region of $(u, v)$ occupied by the initial state, the trajectories associated with the final state can be approximated as straight parallel lines oriented at an angle $\Theta_{f}=\theta_{f} / 2$ from the $u$ axis [Fig. 1(b)]. In this assumption, we 

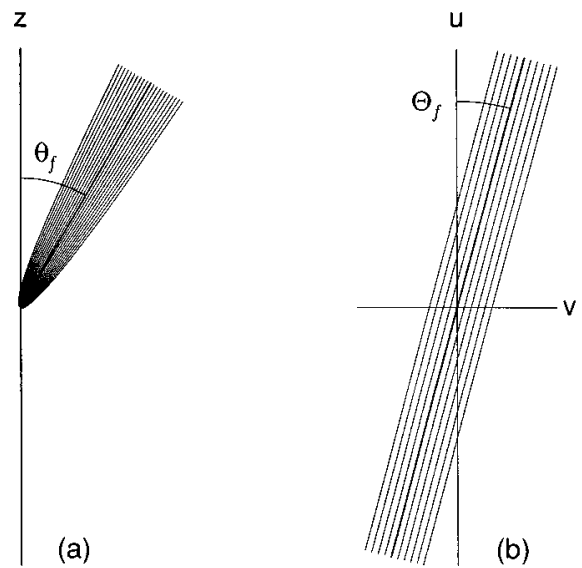

FIG. 1. The family of trajectories representing zero-energy Coulomb scattering near the atomic core in (a) cylindrical and (b) semiparabolic coordinates. The semiparabolic coordinates "rectify" parabolas into straight lines. The symmetry axis for the parabolas determines the incidence angle $\theta_{f}$. Corresponding trajectories in the semiparabolic coordinates make an angle $\Theta_{f}=\theta_{f} / 2$ with the $u$ axis.

are excluding from consideration the possibility that the trajectories may form a caustic near the origin. Such situations are exceptional, and they require special treatment.

(c) Near the nucleus the wave function for the excited state is approximately a zero-energy cylindrically symmetrized Coulomb-scattering wave,

$$
\Psi_{\mathbf{I}}(r, \theta, \phi) \simeq N \Psi_{C, \theta_{f}}^{m}(r, \theta, \phi),
$$

which was defined in the paper of Du and Delos [5], Eqs. (4.22) and (4.23). The incoming part of $\Psi_{C, \theta_{f}}^{m}(r, \theta, \phi)$ is (at $E=0)$

$$
\begin{gathered}
{\left[\Psi_{C, \theta_{f}}^{m}(r, \theta, \phi)\right]_{\mathrm{inc}}} \\
=C_{3} \frac{\exp \left\{-2 i\left(r / a_{0}\right)^{1 / 2}\left[1+\cos \left(\theta-\theta_{f}\right)\right]^{1 / 2}+i m \phi\right\}}{\left(r / a_{0}\right)^{1 / 2}\left(\sin \theta \sin \theta_{f}\right)^{1 / 2} a_{0}^{3 / 2}}, \\
C_{3}=\left(e^{i \pi / 2} / 2^{3 / 2} \pi\right)\left(m_{e} a_{0}^{3} / \hbar^{2}\right) .
\end{gathered}
$$

(d) In $(u, v)$ coordinates, near the nucleus the wave function is approximately a product of Bessel functions; in the case $m=0$,

$$
\begin{aligned}
& \Psi_{\mathbf{I}}(u, v) \simeq N^{\prime} J_{0}\left(p_{u}^{0} u\right) J_{0}\left(p_{v}^{0} v\right), \\
& p_{u}^{0}=2 \cos \Theta_{f}, \quad p_{v}^{0}=2 \sin \Theta_{f} .
\end{aligned}
$$

These assumptions are all equivalent to one another.

At certain points below we will be assuming a one-to-one relationship between $\left(E, \theta_{f}\right)$ and tori: for given $E$, each torus has a unique angle $\theta_{f}$ and each value of $\theta_{f}$ corresponds to a single torus. To understand the meaning of this assumption let us mention some cases in which the assumption does not hold. (i) The torus can be folded in such a way that straight parallel rays approach the origin from two or more directions. For example, in a magnetic field there is symmetry about the $z=0$ plane, and there are tori in which an orbit approaches the origin at an angle $\theta_{f}$ and later at $\pi-\theta_{f}$. (ii) A single quantum state could be associated with two or more tori. For example, again in a magnetic field, because of the $\pm z$ symmetry, there are tori that are localized near the $+z$ and $-z$ axes, having the same values of action variables. Quantum states are superpositions of wave functions associated with these pairs of tori. In such cases, our formula needs modification (Appendix B).

In other papers, we have defined a "recurrence integral"

$$
\left\langle\mathbf{i}|D| \Psi_{C, \theta_{f}}\right\rangle=C_{2} \mathcal{Y}\left(\theta_{f}\right),
$$

where

$$
C_{2}=(4 \pi / \sqrt{2})\left(m_{e} a_{0}^{3} / \hbar^{2}\right)
$$

and $\mathrm{Du}$ and Delos have given formulas for $\mathcal{Y}(\theta)$ \{Eqs. (5.13b), (4.2 and 4.3), and (4.12) in Ref. [5]\}. In closed-orbit theory, we say that the action of the laser on the initial state is to produce near-zero-energy outgoing waves, and $\mathcal{Y}(\theta)$ is their angular distribution.

Combining Eqs. (1.1), (3.1), and (3.6), the oscillator strength is

$$
\begin{aligned}
f_{\mathbf{n}}^{\mathbf{i}} & =\left[2 m_{e}\left(E_{\mathbf{n}}-E_{\mathbf{i}}\right) / \hbar^{2}\right]\left|\left\langle\mathbf{i}|D| \Psi_{\mathbf{I}_{\mathbf{n}}}\right\rangle\right|^{2} \\
& =\left[2 m_{e}\left(E_{\mathbf{n}}-E_{\mathbf{i}}\right) / \hbar^{2}\right]\left|\left\langle\mathbf{i}|D| N_{\mathbf{n}} \Psi_{C, \theta_{f}(\mathbf{n})}^{m}\right\rangle\right|^{2} \\
& =\left[2 m_{e}\left(E_{\mathbf{n}}-E_{\mathbf{i}}\right) / \hbar^{2}\right]\left|N_{\mathbf{n}}\right|^{2} C_{2}^{2}\left|\mathcal{Y}\left(\theta_{f}(\mathbf{n})\right)\right|^{2} .
\end{aligned}
$$

To complete our evaluation, we only have to find the coefficient $N_{\mathrm{n}}$. This we do by matching the quantum form near the nucleus to the semiclassical form at larger distances.

\section{MATCHING SEMICLASSICAL AND QUANTUM FORMS}

We find $N$ by equating

$$
\begin{aligned}
N \Psi_{C, \theta_{f}}^{m}(r, \theta, \phi)= & (2 \pi)^{-3 / 2} \sum_{j} J_{r \theta}^{1 / 2}\left|\frac{\partial^{2} S_{j}\left(r, \theta, I_{1}, I_{2}\right)}{\partial(r, \theta) \partial\left(I_{1}, I_{2}\right)}\right|^{1 / 2} \\
& \times \exp \left\{i\left[S_{j}\left(r, \theta, I_{1}, I_{2}\right) / \hbar+m \phi-\mu_{j} \pi / 2\right]\right\}
\end{aligned}
$$

in a region far enough from the nucleus that the semiclassical form is valid, but close enough that the approximate quantum form is valid (we substituted $L_{z}$ by its quantized value $m \hbar)$. It is most appropriate to match these expressions on the line $\theta=\theta_{f}$ at a moderate distance from the atom.

For this purpose we need the form of $S\left(r, \theta, I_{1}, I_{2}\right)$ : specifically we need its dependence on $\left(r, \theta, I_{1}, I_{2}\right)$ so that we can evaluate the mixed derivatives. We already assumed that the transformation $\left(I_{1}, I_{2}\right) \leftrightarrow\left(E, \theta_{f}\right)$ is invertible, so we may use $S\left(r, \theta, E, \theta_{f}\right)$. From our assumption (a) about the trajectories, one can show that near $E \sim 0, \theta \sim \theta_{f}$, for the incoming term in the wave function, 


$$
\begin{aligned}
S\left(r, \theta, E, \theta_{f}\right)= & -\left\{2\left(r / a_{0}\right)^{1 / 2}+\frac{1}{3}\left(m a_{0}^{2} E / \hbar^{2}\right)\left(r / a_{0}\right)^{3 / 2}\right. \\
& \left.\times\left[2-\cos \left(\theta-\theta_{f}\right)\right]\right\}\left[1+\cos \left(\theta-\theta_{f}\right)\right]^{1 / 2} \hbar .
\end{aligned}
$$

This formula is similar to the phase in Eq. (3.2), but the present formula contains an additional term, $\frac{1}{3} \operatorname{Er}^{3 / 2}[2$ $\left.-\cos \left(\theta-\theta_{f}\right)\right]$, which is needed so that we can evaluate $\partial S / \partial E$.

The determinant of the second derivatives entering Eq. (4.1) for the wave function can now be found with the use of Eq. (4.2) and the transformation rule

$$
\begin{gathered}
\frac{\partial^{2} S_{j}\left(r, \theta, I_{1}, I_{2}\right)}{\partial(r, \theta) \partial\left(I_{1}, I_{2}\right)}=\frac{\partial^{2} S_{j}(r, \theta, E, \beta)}{\partial(r, \theta) \partial(E, \beta)} \frac{\partial(E, \beta)}{\partial\left(I_{1}, I_{2}\right)}, \\
\frac{\partial^{2} S_{j}(r, \theta, E, \beta)}{\partial(r, \theta) \partial(E, \beta)}=-\frac{m_{e} r}{2 \sin \theta_{f}} \frac{\partial \cos \theta_{f}}{\partial \beta} .
\end{gathered}
$$

This way the incoming part of Eq. (4.1) is (at $E=0$ )

$$
\begin{aligned}
N \Psi_{C, \theta_{f}}^{m}(r, \theta, \phi)= & \frac{e^{-i \mu_{j} \pi / 2}}{\sqrt{2 \pi}} \sqrt{\left|\frac{\partial(E, \beta)}{\partial\left(I_{1}, I_{2}\right)}\right|} \sqrt{\left|\frac{\partial \cos \theta_{f}}{\partial \beta}\right|} \\
& \times \frac{m_{e}}{2^{3 / 2} \pi} \frac{e^{-i 2\left\{r\left[1+\cos \left(\theta-\theta_{f}\right)\right]\right\}^{1 / 2}}}{\sqrt{r \sin \theta} \sqrt{\sin \theta_{f}}} e^{i m \phi} .
\end{aligned}
$$

Comparison with the expression for $\Psi_{C, \theta_{f}}^{m}(r, \theta, \phi)$ [Eq. (3.2)] tells us that

$$
|N|=\frac{1}{\sqrt{2 \pi}} \sqrt{\left|\frac{\partial(E, \beta)}{\partial\left(I_{1}, I_{2}\right)}\right|} \sqrt{\left|\frac{\partial \cos \theta_{f}}{\partial \beta}\right|}\left(\hbar^{2} / m_{e}^{1 / 2} a_{0}^{2}\right) .
$$

With use of the definition (3.6) for the angular distribution $\mathcal{Y}\left(\theta_{f}\right)$ and expression (4.6) for $|N|$, the formula (3.8) transforms into the final result

$$
f_{\mathbf{n}}^{\mathbf{i}}=\frac{8 \pi m_{e}^{2} a_{0}^{2}\left(E_{\mathbf{n}}-E_{\mathbf{i}}\right)}{\hbar^{2}}\left|\frac{\partial(E, \beta)}{\partial\left(I_{1}, I_{2}\right)}\right|\left|\frac{\partial \cos \theta_{f}}{\partial \beta}\right|\left|\mathcal{Y}\left(\theta_{f}(\mathbf{n})\right)\right|^{2}
$$

Some generalizations of the above procedure are described in Appendix B.

In many cases we can just take $\beta=\cos \theta_{f}$. That holds in the particular case of a hydrogen atom in an external electric field, and we get the formula considered in our previous paper [1]:

$f_{\mathbf{n}}^{\mathbf{i}}=\frac{8 \pi m_{e}^{2} a_{0}^{2}\left(E_{\mathbf{n}}-E_{\mathbf{i}}\right)}{\hbar^{2}}\left|\frac{\partial\left(E, \cos \theta_{f}(\mathbf{n})\right)}{\partial\left(I_{1}, I_{2}\right)}\right|\left|\mathcal{Y}\left(\theta_{f}(\mathbf{n})\right)\right|^{2}$.

The value of this expression is to be taken at the eigenejection angle, which is given by the Bohr-Sommerfeld quantization conditions (2.3).

\section{SMOOTH BACKGROUND ABSORPTION IN THE STARK SYSTEM}

The above formula applies to discrete quantum states. Right at the beginning, we assumed that the trajectories form

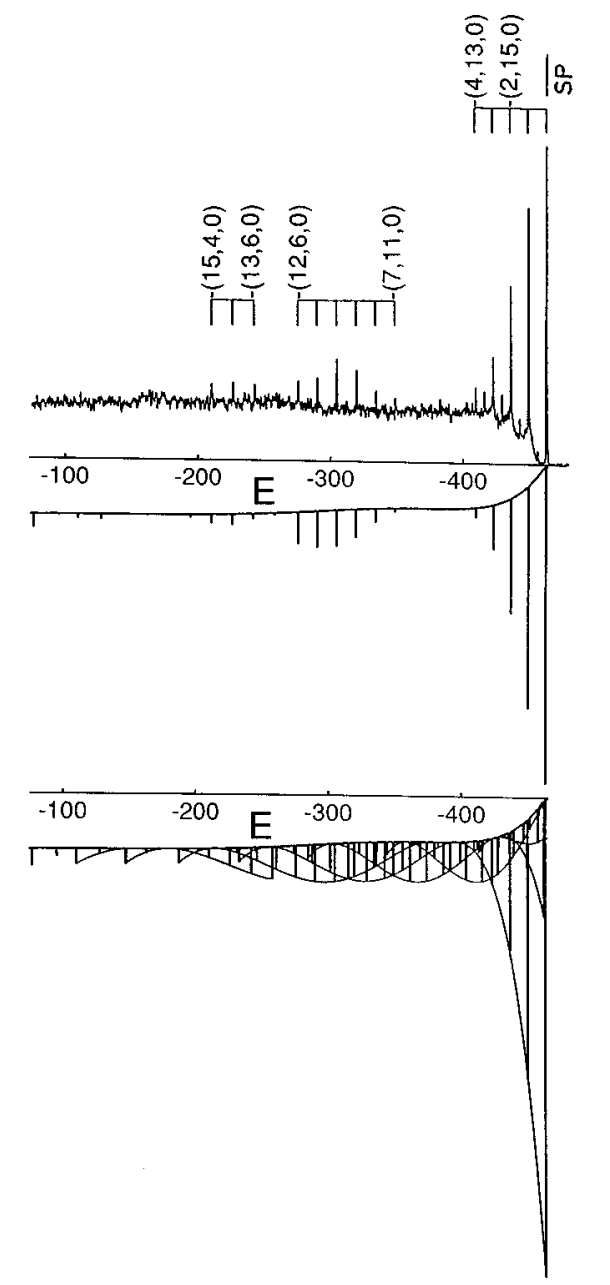

(a)

(b)

(c)

FIG. 2. The result of modeling the experimental photoabsorption spectrum of hydrogen in an external electric field $F$ $=5714 \mathrm{~V} / \mathrm{cm}$ by semiclassical formula (4.8) with background given by Eq. (5.7) (mirror plot). The initial parabolic state $\left(n_{1}, n_{2}, m\right)$ $=(0,1,0)$ is excited by parallel linearly polarized radiation $(\pi$ polarization). The energy is given in $\mathrm{cm}^{-1}$. We show the part of the spectrum above the saddle point (SP) energy $E_{s}=-463 \mathrm{~cm}^{-1}$. The numbers $\left(n_{1}, n_{2}, m\right)$ in the upper part of the plot identify some parabolic final states. (a) Experiment, from Ref. [2]. (b) Semiclassical calculation adjusted according to the experimental signal discrimination time and the laser bandwidth (see text for details). (c) Semiclassical calculations, with envelopes of Stark manifolds.

tori, i.e., the electron has regular orbits in a bounded region of configuration space; therefore the corresponding quantum spectrum is discrete.

In the Stark system, the energy spectrum is, in principle, continuous over the whole energy axis, $-\infty<E<\infty$. In reality, below the saddle energy $E_{s}=-2 F^{1 / 2}$, we have a quasidiscrete spectrum - each energy level is broadened because the electron can tunnel through the barrier. Nevertheless there is a well-defined classically allowed region; all the other assumptions that went into our derivation are applicable, and therefore the energy $E_{\mathbf{n}}$ of each quasidiscrete level can be calculated by quantization of action, and the oscillator strength of that level is given by Eq. (4.8).

For energies above $E=0$, all electrons directly escape, and the absorption spectrum is continuous. For energies between $E_{s}$ and zero, it is appropriate to think of the absorption 


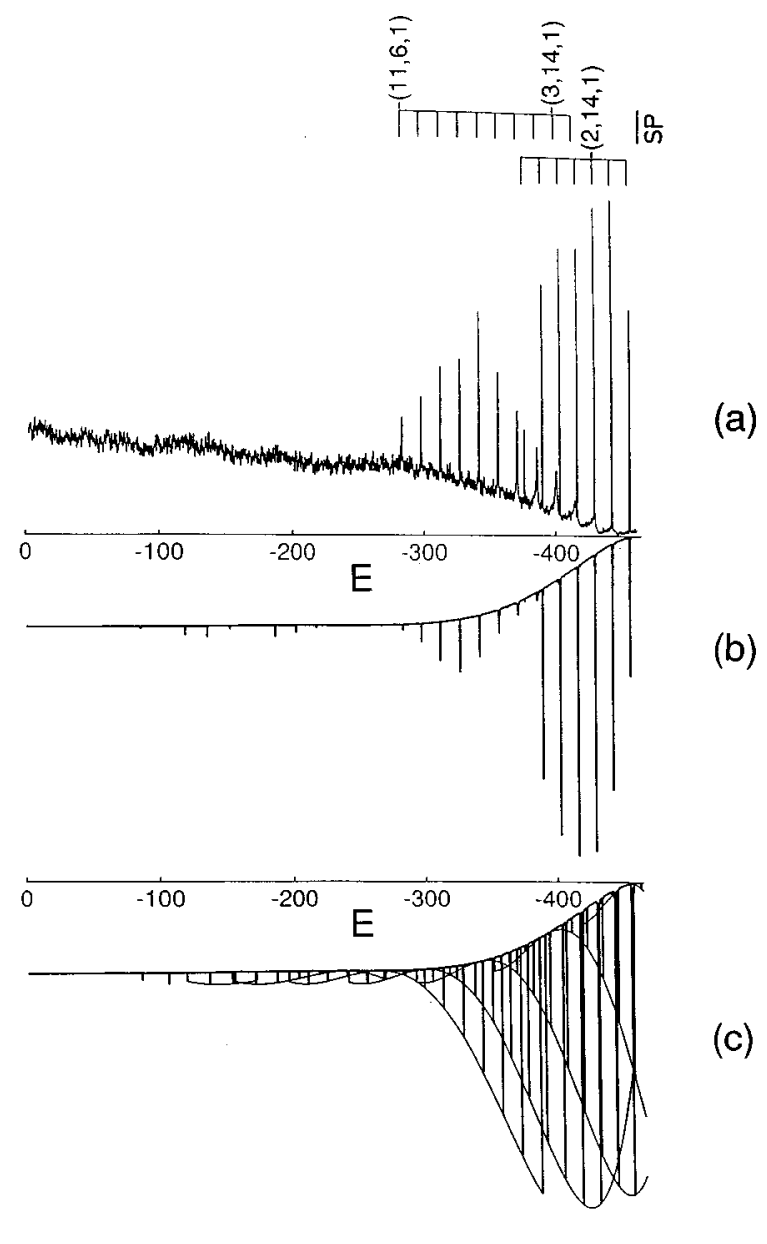

FIG. 3. The same as in Fig. 2 for ionization by $\sigma$-polarized radiation. Note the difference in the shape of the envelopes in comparison with Fig. 2, and the different behavior of the background.

spectrum as a superposition of a continuous and a quasidiscrete spectrum. This is immediately seen by looking at the experimental measurements shown in Figs. 2 and 3.

Closer inspection shows that the continuous spectrum has some structure on the largest scales. In Fig. 2, going from right to left, we see a sharp rise in continuum absorption at $E=-500 \mathrm{~cm}^{-1}$ followed by a plateau near $E=-350 \mathrm{~cm}^{-1}$ and then another gentle rise around $E=-275 \mathrm{~cm}^{-1}$ to a second plateau (use a straightedge to see this). In Fig. 3, the low-energy rise is more gentle, but the final value is larger than in Fig. 2. How do we explain this structure?

Classical trajectory calculations show that there is a critical angle $\theta_{c}=\theta_{c}(E, F)$ such that if the electron leaves the atom at angles between $\theta_{c}$ and $\pi$, the electron escapes. However, if it leaves the atom with $0<\theta<\theta_{c}$, the electron remains in a bound quasiperiodic orbit. Intuitively we might expect that those classically escaping paths would correspond to the continuous absorption, while the classically bound ones would correspond to absorption to quasidiscrete states. Indeed, Eq. (1.3) says that the oscillator-strength density for the continuum is proportional to the integral of $|\mathcal{Y}(\theta)|^{2}$, the angular distribution of outgoing electrons, over the escape sector. Let us now derive this formula.

Our derivation again involves assumptions that are somewhat more general than the Stark problem. We assume the existence of a second conserved quantity $\beta$, which is, in prin- ciple, observable (i.e., $\beta$ is "an observable" according to the rules of quantum mechanics). We assume that at each $E$ there is a range of $\beta\left[\beta_{1}(E)<\beta<\beta_{2}(E)\right]$ for which trajectories escape, and a complementary range for which trajectories are bound. We assume that there is a one-to-one relationship between $\beta$ and the ejection angle $\theta_{f}$. Hence there is a range of $\theta_{f}\left[\theta_{1}(E)<\theta_{f}<\theta_{2}(E)\right]$ leading to escape.

Instead of working with the individual oscillator strengths $f_{\mathbf{n}}^{\mathbf{i}}$, let us work with the "integrated oscillator strength," $f(E)$, i.e., the oscillator strength summed over all levels having energy less than $E$ :

$$
f(E)=\sum_{\left\{\mathbf{n} \mid E_{\mathbf{n}}<E\right\}} f_{\mathbf{n}}^{\mathbf{i}}
$$

This is defined such that

$$
\begin{gathered}
\lim _{E \rightarrow-\infty} f(E)=0, \quad \lim _{E \rightarrow \infty} f(E)=1, \\
d f(E) / d E \equiv D f(E)=\sum_{\mathbf{n}} f_{\mathbf{n}}^{\mathbf{i}} \delta\left(E-E_{\mathbf{n}}\right) .
\end{gathered}
$$

$f(E)$ has steps at each energy level. When we go from the discrete to the continuous limit, $f(E)$ becomes a continuous monotonically increasing function of $E$ with $0<f(E)<1$ [6].

Suppose at each energy we have identified the trajectories for which the electron escapes. For each $\left(E, \theta_{f}\right)$ there is a corresponding $(E, \beta)$, and we can define corresponding action variables $\left(I_{1}, I_{2}\right)$ for those trajectories, for example, by confining the trajectories to an appropriate box. By quantizing in this box, we get a correspondence between action variables and quantum states $\mathbf{I}=(\mathbf{n}+1 / 2) \hbar$. Then the integrated oscillator strength corresponding to escaping trajectories is

$$
\begin{aligned}
f_{\text {escape }}(E)= & \sum_{\substack{\mathbf{n} \text { escaping } \\
E_{\mathbf{n}}<E}} 8 \pi\left(m_{e}^{2} a_{0}^{2} / \hbar^{2}\right)\left(E_{\mathbf{n}}-E_{\mathbf{i}}\right) \\
& \times\left|\frac{\partial(E, \beta)}{\partial\left(I_{1}, I_{2}\right)} \frac{\partial \cos \theta}{\partial \beta}\right|\left|\mathcal{Y}\left(\theta_{\mathbf{n}}\right)\right|^{2} \\
= & \sum_{\substack{\mathbf{n} \text { escaping } \\
E_{\mathbf{n}}<E}} 8 \pi\left(m_{e}^{2} a_{0}^{2} / \hbar^{2}\right)\left(E_{\mathbf{n}}-E_{\mathbf{i}}\right) \\
& \times\left.\left|\frac{\partial(E, \beta)}{\partial\left(I_{1}, I_{2}\right)} \frac{\partial \cos \theta}{\partial \beta}\right| \mathcal{Y}\left(\theta_{\mathbf{n}}\right)\right|^{2} \\
& \times\left|\frac{\Delta\left(n_{1}, n_{2}\right)}{\Delta\left(I_{1}, I_{2}\right)} \frac{\Delta\left(I_{1}, I_{2}\right)}{\Delta(E, \beta)}\right| \Delta(E, \beta) \\
& \rightarrow \int_{-\infty}^{E} d E^{\prime} \int_{\beta_{1}(E)}^{\beta_{2}(E)} d \beta(8 \pi)\left(m_{e}^{2} a_{0}^{2} / \hbar^{2}\right)\left(E^{\prime}-E_{\mathbf{i}}\right) \\
& \times\left.\left|\frac{\partial\left(E^{\prime}, \beta\right)}{\partial\left(I_{1}, I_{2}\right)} \frac{\partial \cos \theta}{\partial \beta}\right| \mathcal{Y}\left(\theta_{\mathbf{n}}\right)\right|^{2}\left|\frac{\partial\left(n_{1}, n_{2}\right)}{\partial\left(I_{1}, I_{2}\right)} \frac{\partial\left(I_{1}, I_{2}\right)}{\partial\left(E^{\prime}, \beta\right)}\right|
\end{aligned}
$$

The $\Delta$ quantities $\Delta\left(n_{1}, n_{2}\right) / \Delta\left(I_{1}, I_{2}\right)$ are discrete analogues of the corresponding Jacobians, and in going from (5.3b) to 
(5.3c) we have gone to the continuum limit. Now $|\partial \mathbf{n} / \partial \mathbf{I}|$ $=\hbar^{-2}$, and almost everything else in $(5.3 \mathrm{c})$ cancels, leaving only

$$
\begin{aligned}
f(E)= & \int_{-\infty}^{E} d E^{\prime} \int_{\beta_{1}\left(E^{\prime}\right)}^{\beta_{2}\left(E^{\prime}\right)} d \beta(8 \pi)\left(m_{e}^{2} a_{0}^{2} / \hbar^{4}\right) \\
& \times\left(E^{\prime}-E_{\mathbf{i}}\right)|\mathcal{Y}(\theta)|^{2}\left|\frac{\partial \cos \theta}{\partial \beta}\right| \\
= & \int_{-\infty}^{E} d E^{\prime} \int_{\theta_{1}\left(E^{\prime}\right)}^{\theta_{2}\left(E^{\prime}\right)} 8 \pi\left(m_{e}^{2} a_{0}^{2} / \hbar^{4}\right) \\
& \times\left(E^{\prime}-E_{\mathbf{i}}\right)|\mathcal{Y}(\theta)|^{2} \sin \theta d \theta
\end{aligned}
$$

and therefore

$$
\begin{aligned}
D f(E) & =d f(E) / d E \\
& =8 \pi\left(m_{e}^{2} a_{0}^{2} / \hbar^{4}\right)\left(E-E_{\mathbf{i}}\right) \int_{\theta_{1}(E)}^{\theta_{2}(E)}|\mathcal{Y}(\theta)|^{2} \sin \theta d \theta .
\end{aligned}
$$

In this final formula, the (box-quantized) action variables for free orbits have disappeared in favor of quantities that are well defined.

The final formula has a clear physical meaning: the intensity of the smooth background in absorption spectra is proportional to the total flux along those trajectories which lead from the atomic core to infinity. We will compare this formula with experiments in the following section. For the Stark problem

$$
\begin{gathered}
\theta_{1}(E)=\theta_{c}=\cos ^{-1}\left(1-E^{2} / 2 F\right), \\
\theta_{2}(E)=\pi,
\end{gathered}
$$

so we rewrite Eq. (5.5) in the form

$$
D f(E)=8 \pi\left(m_{e}^{2} a_{0}^{2} / \hbar^{4}\right)\left(E-E_{\mathbf{i}}\right) \int_{0}^{\alpha_{c}} I(\alpha) d \alpha
$$

with

$$
I(\alpha)=|\mathcal{Y}(\pi-\theta)|^{2} \sin (\pi-\theta), \quad \alpha_{c}=\pi-\theta_{c} .
$$

\section{COMPARISON WITH EXPERIMENTS AND NUMERICAL CALCULATIONS}

As was shown in our preliminary communication [1], the semiclassical formula (4.8) for quasidiscrete states is in excellent agreement with quantum calculations of oscillator strengths. In that paper, we considered the hydrogen atom in an electric field $F$ with energies $E$ below the saddle point $E_{s}=-2 \sqrt{F}$. In another paper [7] we compared this formula with experimental measurements of quasidiscrete levels of the $\mathrm{Li}$ atom in an electric field at energies above the saddle. Again good agreement was obtained.

In the present paper we compare this semiclassical formula with the experiment of Rottke and Welge [2], who measured photoabsorption of a hydrogen atom in an electric field in the region above the saddle. The upper parts of Figs. 2 and 3 show their experimental results. In both cases, the hydrogen atom was first excited to the $\left(n_{1}=0, n_{2}=1, m\right.$ $=0)$ Stark state (the lowest-energy state at $n=2$ ), and then it was further excited to states around $n=17$ and higher. The applied electric field $F$ was $5714 \mathrm{~V} / \mathrm{cm}$, giving a saddle energy $E_{s}=-463 \mathrm{~cm}^{-1}$. In Fig. 2, the laser was linearly polarized parallel $(\pi)$ to $F$, while in Fig. 3 it was linearly polarized perpendicular $(\sigma)$ to $F$.

Our most complete semiclassical results are shown in Figs. 2(c) and 3(c). We take the experimental spectrum to be the sum of the quasidiscrete spectrum, calculated by the semiclassical formula (4.8), plus the background term (5.7), which is proportional to the total flux along classical trajectories going from the atom to infinity.

For energies between -500 and $-300 \mathrm{~cm}^{-1}$, there is a large number of quasidiscrete states. The absorption spectrum does not show any clear pattern until we identify the overlapping $n$ manifolds. Then we can see that the oscillator strengths of the quasidiscrete states follow the pattern dictated by $|\mathcal{Y}(\theta(\mathbf{n}))|^{2}$, as indicated by the envelopes shown in Figs. 2-4. (Two effects on the absorption spectrum have not been considered in these calculations. We excluded broad above-barrier resonances, and we did not incorporate the natural widths of any quasidiscrete state. Each of these has a small but visible effect on the absorption spectrum.)

To compare with the measurements, two experimental factors must be considered, as emphasized in [2]. (1) Quasidiscrete states having lifetimes greater than about $2 \times 10^{-6}$ sec were not detected. (2) The ratio of laser linewidth to the natural width of an absorption varies widely over the spectrum. When the laser width is larger than the natural width, the observed peak will be lower and wider than the calculated peak.

To account for (1), we cut off all long-lived quasidiscrete states, using a factor $\left\{1+\exp \left[\alpha\left(t / t_{0}-1\right)\right]\right\}^{-1}$ with $t_{0}$ $=10^{-6} \mathrm{sec}$ and $\alpha=5$. Still, most of the peaks that remain have widths that are less than the laser linewidth. To account for (2), we "renormalized" the theoretical peak-tobackground ratio such that the one largest peak in Fig. 2 and the smooth background at $E \approx 0$ would match the experiment. Finally, we again neglected the broad above-barrier resonances. The same renormalization constant for background was then used to draw Fig. 3.

These two modifications of the semiclassical theory give results shown in Figs. 2(b) and 3(b). Quite respectable agreement with experiment is found, again helping to confirm the validity of the semiclassical formulas.

The combination plot, Fig. 4, shows how the semiclassical formula (5.7) describes the smooth background spectrum in the conditions of this experiment. The critical angle of ejection dividing bound from free trajectories depends only on the scaled energy $\varepsilon=E / \sqrt{F}=2 E / E_{s}$ [Eq. (5.6)],

$$
\theta_{c}=\cos ^{-1}\left(1-\frac{1}{2} \varepsilon^{2}\right)
$$

and in the lower-right portion of Fig. 4 we plot $\alpha_{c}(\varepsilon)=\pi$ $-\theta_{c}(\varepsilon)$ versus $\varepsilon$ for $\varepsilon$ between -2 and 0 .

The angular distribution of outgoing waves is plotted versus $\alpha=\pi-\theta$ in the lower-left portion of Fig. 4. Initial states in this system are the very unsymmetrical $n=2$ hydrogenic Stark states, and they lead to the characteristic angular distributions shown. Curve 2 is the one associated with the ex- 


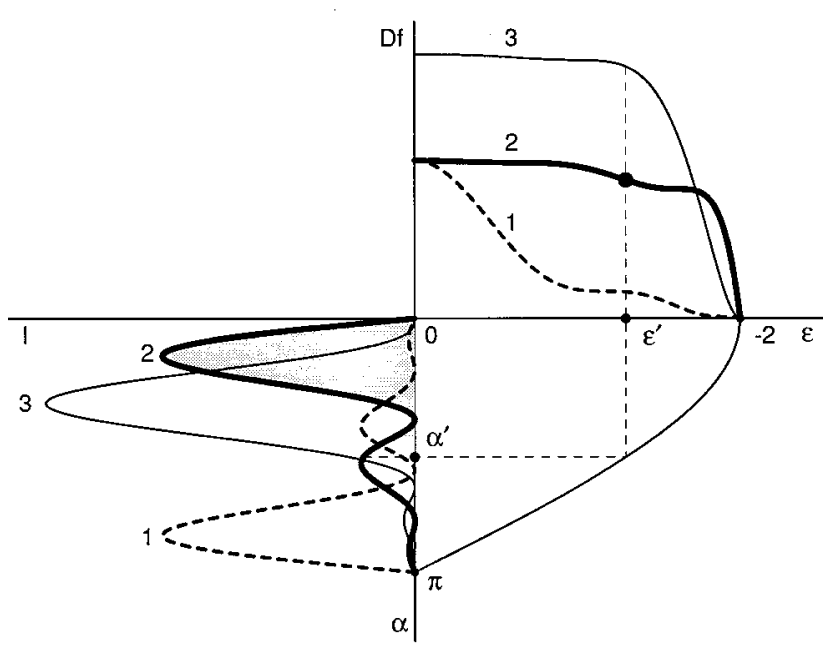

FIG. 4. Combination plot showing the calculation of continuum absorption in Stark spectra according to semiclassical formula (5.7). The dependence of the critical angle $\alpha_{c}$ on the scaled energy $\varepsilon$ $=E / \sqrt{F}$ [Eqs. (5.8) and (5.6)] is shown in the lower right part of the plot. On the lower left is plotted the angular distribution of outgoing waves $I(\alpha)$ vs $\alpha$ for three cases. Curve 1: $\left(n_{1}, n_{2}, m\right)=(1,0,0)$ initial state excited by linear parallel $(\pi)$ radiation. Curve 2: $(0,1,0)$ initial state excited by $\pi$-polarized radiation. Curve $3:(0,1,0)$ initial state excited by linear perpendicular $(\sigma)$ radiation. The predicted continuum absorption $D f(E)$ from Eq. (5.7) at scaled energy $\varepsilon^{\prime}$ is proportional to the integral of $I(\alpha)$ from zero to $\alpha^{\prime} \equiv \alpha_{c}\left(\varepsilon^{\prime}\right)$ (shaded area). These integrals are shown as functions of $\varepsilon$ in the upper right. Curves 2 and 3 correspond, respectively, to experiments shown in Figs. 2 and 3.

periment shown in Figs. 2. The background absorption is proportional to the area under the curve (shaded area). Thus in this case the background should rise quickly to a plateau [the center of which corresponds to the node of $I(\alpha)$ ], and then it should have a second small rise to its final value. This is precisely what we saw in Fig. 2. Curve 3 in Fig. 4 has a more gradual rise to a larger final value, as in Fig. 3 .

The converse of the above is the easier statement: the derivative of the smooth background $d[D f(E)] / d E$ plotted as a function of $\theta_{c}=\cos ^{-1}\left(1-E^{2} / 2 F\right)$ is a graph of $|\mathcal{Y}(\theta)|^{2} \sin \theta$.

\section{CONCLUSION}

We give a semiclassical formula for the oscillator strength of discrete and continuous regular spectra in external fields. The formula holds under our major assumption that the electron trajectories are regular, and that near the atom they are similar to those for zero-energy Coulomb scattering.

As explained in Ref. [1], this formula combines classical, semiclassical, and quantum concepts to obtain the oscillator strength. (Quantum) $\mathcal{Y}\left(\theta_{f}\right)$ is the quantum angular distribution of outgoing waves for a zero-energy electron that had been excited from the state i. (Semiclassical) each discrete quantum state is connected to an eigentrajectory through the semiclassical quantization conditions (2.3). (Classical) each such eigentrajectory has (it is assumed) one classical angle $\theta_{f}$, the angle of ejection of the electron from the atom. The oscillator strength to the state $\mathbf{n}$ is proportional to the angular distribution of outgoing waves $\left|\mathcal{Y}\left(\theta_{f}\right)\right|^{2}$ at that classical angle $\theta_{f}(\mathbf{n})$, and the oscillator strength density in the con- tinuum is proportional to the integral of $|\mathcal{Y}(\theta)|^{2}$ over the escape sector.

The other factor in the formula,

$$
\left|\frac{\partial\left(E, \cos \theta_{f}\right)}{\partial\left(I_{1}, I_{2}\right)}\right|=\left|\frac{\partial\left(I_{1}, I_{2}\right)}{\partial\left(E, \cos \theta_{f}\right)}\right|^{-1},
$$

is the inverse of the density of eigentrajectories in energy and outgoing angle. This factor is related to the large-scale structure of the tori.

\section{ACKNOWLEDGMENTS}

This work was supported by the National Science Foundation. We thank M. Haggerty for helpful discussions, and S. Freund and H. Rottke for the print-quality experimental plots.

\section{APPENDIX A: PROOF OF NORMALIZATION}

Here we will prove that the wave function $\Psi_{\mathrm{I}}(\mathbf{q})$ [Eq. (2.4)] is normalized to unity in the case when the corresponding Lagrangian manifold is a torus (the motion is finite).

When integrating $\left|\Psi_{\mathbf{I}}(\mathbf{q})\right|^{2}$, we can neglect the cross terms because they are oscillating and do not give significant contribution to the normalization integral. So we can write

$$
\int_{D} d \mathbf{q}\left|\Psi_{\mathbf{I}}(\mathbf{q})\right|^{2}=(2 \pi)^{-k} \sum_{j} \int_{D} d \mathbf{q}\left|\left(\partial^{2} S_{j}(\mathbf{q}, \mathbf{I}) / \partial \mathbf{q} \partial \mathbf{I}\right)\right|,
$$

where $D$ denotes the classically accessible region in coordinate space.

As $S$ [Eq. (2.2)] is a type-2 generating function, $\partial S / \partial \mathbf{I}$ $=\boldsymbol{\phi}, \boldsymbol{\phi}$ being the angle coordinates corresponding to the actions I, we can rewrite (A1) in the form

$$
\int_{D} d \mathbf{q}\left|\Psi_{\mathbf{I}}(\mathbf{q})\right|^{2}=(2 \pi)^{-k} \sum_{j} \int_{D} d \mathbf{q}|\partial(\boldsymbol{\phi}) / \partial(\mathbf{q})| .
$$

As the torus is an oriented manifold, all members of the sum (A2) combine in one angular integral over torus $T$ :

$$
\int_{D} d \mathbf{q}\left|\Psi_{\mathbf{I}}(\mathbf{q})\right|^{2}=(2 \pi)^{-k} \int_{T} d \boldsymbol{\phi}=(2 \pi)^{-k}(2 \pi)^{k}=1 .
$$

\section{APPENDIX B: GENERALIZATIONS OF THE SEMICLASSICAL FORMULA}

The derivation of the semiclassical formula for oscillator strengths can easily be modified to treat more complex systems. For example, we may consider systems without axial symmetry (three-dimensional motion of the active electron). One can also incorporate some internal degrees of freedom if a more complex object than the hydrogen atom is considered (for example, photoabsorption by molecules in external fields).

Let us consider the case of three-dimensional motion of the photoelectron. In this case we assume the existence of three action variables $\mathbf{I}=\left(I_{1}, I_{2}, I_{3}\right)$ or another triplet of conserved quantities, say, $(E, \beta, \gamma)$ (as before, $\beta$ and $\gamma$ are sup- 
posed to be dimensionless). In the vicinity of the atomic core we can consider a family of zero-energy Coulomb trajectories, which is defined now by two angular parameters $\left(\theta_{f}, \phi_{f}\right)$. This means that there is an orbit that comes to the nucleus on a straight line from the direction $\left(\theta_{f}, \phi_{f}\right)$. Other orbits in the family are parabolas centered on this line and the family of parabolas is cylindrically symmetric about this line. We assume a one-to-one correspondence between the conserved quantities $(\beta, \gamma)$ and the returning direction $\left(\theta_{f}, \phi_{f}\right)$.

Next we can introduce a "Coulomb-reference" function $\Psi_{C, \theta_{f}, \phi_{f}}(r, \theta, \phi)$ - the same as that introduced in Eq. (4.18a) of [5], except for a normalizing factor $(2 \pi)^{-1 / 2}$,

$$
\begin{aligned}
\Psi_{C, \theta_{f}, \phi_{f}}(r, \theta, \phi) & =(2 \pi)^{-1 / 2} \Psi_{C, \theta_{f}, \phi_{f}}^{[5]}(r, \theta, \phi) \\
& =-i C_{3} \frac{\exp \left(-i 2\left\{\left(r / a_{0}\right)\left[1+\cos \theta \cos \theta_{f}+\sin \theta \sin \theta_{f} \cos \left(\phi-\phi_{f}\right)\right]\right\}^{1 / 2}\right)}{\left\{\left(r / a_{0}\right)\left[1+\cos \theta \cos \theta_{f}+\sin \theta \sin \theta_{f} \cos \left(\phi-\phi_{f}\right)\right]\right\}^{1 / 4} a_{0}^{3 / 2}}
\end{aligned}
$$

[the coefficient $C_{3}$ is defined in Eq. (3.3)]. Comparable to assumption (e) in the Introduction, we assume that the threedimensional semiclassical wave function (2.4) behaves like Eq. (B2) in the zone of Coulomb dominance,

$$
\Psi_{\mathbf{I}}(r, \theta, \phi)=N \Psi_{C, \theta_{f}, \phi_{f}}(r, \theta, \phi)
$$

Then by the method used to obtain Eq. (4.6), we find

$$
\begin{aligned}
N & =\frac{e^{-i \mu_{j} \pi / 2}}{\sqrt{2 \pi}} \sqrt{\left|\frac{\partial(E, \beta, \gamma)}{\partial\left(I_{1}, I_{2}, I_{3}\right)}\right|} \sqrt{\left|\frac{\partial\left(\cos \theta_{f}, \phi_{f}\right)}{\partial(\beta, \gamma)}\right|} \frac{\hbar^{5 / 2}}{m_{e}^{1 / 2} a_{0}^{2}} \\
& =\frac{e^{-i \mu_{j} \pi / 2}}{\sqrt{2 \pi}} \sqrt{\left|\frac{\partial\left(E, \cos \theta_{f}, \phi_{f}\right)}{\partial\left(I_{1}, I_{2}, I_{3}\right)}\right|} \frac{\hbar^{5 / 2}}{m_{e}^{1 / 2} a_{0}^{2}} .
\end{aligned}
$$

This way we come to an analog of Eq. (4.7) for the oscillator strength:

$$
\begin{aligned}
f_{\mathbf{n}}^{\mathbf{i}}= & \frac{8 \pi m_{e}^{2} a_{0}^{2}\left(E_{\mathbf{n}}-E_{\mathbf{i}}\right)}{\hbar}\left|\frac{\partial(E, \beta, \gamma)}{\partial\left(I_{1}, I_{2}, I_{3}\right)}\right| \\
& \times\left.\left|\frac{\partial\left(\cos \theta_{f}, \phi_{f}\right)}{\partial(\beta, \gamma)}\right| \mathcal{Y}\left(\theta_{f}, \phi_{f}\right)\right|^{2}
\end{aligned}
$$

with

$$
\mathcal{Y}\left(\theta_{f}, \phi_{f}\right)=C_{2}^{-1}\left\langle\mathbf{i}|D| \Psi_{C, \theta_{f}, \phi_{f}}\right\rangle,
$$

which again also represents the angular distribution of ejected electrons.

Another case can also arise in either two or three dimensions. An eigentorus could be folded in phase space in such a way that orbits return to the nucleus from several distinct directions $\left(\theta_{f}^{l}(\mathbf{n}), \phi_{f}^{l}(\mathbf{n})\right)$, where $l=1,2, \ldots$, labels the discrete directions from which orbits return to the atom, and $\mathbf{n}$ again labels the eigentorus. Alternatively, in a system having symmetry, a given quantum state may correspond to a superposition of wave functions associated with two or more eigentori. For example, if the system is symmetric under $z \rightarrow-z$, there may be distinct returning orbits near $+z$ and $-z$ axes.

Such cases are characterized by superposition and interference of waves associated with several closed trajectories. In this case, the wave function of the final state is represented by the sum of addends in the form (3.1) for all contributing tori, and the oscillator strength has the form

$f_{\mathbf{n}}^{\mathbf{i}}=\frac{16 \pi^{2} m_{e}^{3} a_{0}^{6}\left(E_{\mathbf{n}}-E_{\mathbf{i}}\right)}{\hbar^{6}}\left|\sum_{l} N_{\mathbf{n}}^{(l)} \mathcal{Y}\left(\theta_{f}^{(l)}(\mathbf{n}), \phi_{f}^{(l)}\right)\right|^{2}$,

where the sum is taken over all ejection angles and $N_{\mathbf{n}}^{(l)}$ is given by Eq. (B4).

We should note that the presence of a Coulomb field in the final state is also not essential. We can think, for example, about a negative ion in a cavity. In this case the final state belongs to the discrete spectrum, but only a short-range interaction of the active electron with the atom is present. In this case the reference function is a plane wave labeled with the constants of free motion.

In the case when the core possesses some additional degrees of freedom, the function $\Psi_{C, \theta_{f}, \phi_{f}}$ may be entangled with them.
[1] V. Kondratovich and J. B. Delos, Phys. Rev. A 56, R5 (1997).

[2] H. Rottke and K. H. Welge, Phys. Rev. A 33, 301 (1986).

[3] W. H. Miller, J. Chem. Phys. 53, 1949 (1970).

[4] R. G. Littlejohn, J. Math. Phys. 31, 2952 (1990).

[5] M. L. Du and J. B. Delos, Phys. Rev. A 38, 1896 (1988); 38, 1913 (1988).
[6] In contrast, in this limit $f_{\mathbf{n}}^{\mathbf{i}}$ goes to zero while the density of states goes to infinity. Therefore $f(E)$ is mathematically easier to work with.

[7] N. Spellmeyer, D. Kleppner, M. R. Haggerty, V. Kondratovich, J. B. Delos, and J. Gao, Phys. Rev. Lett. 79, 1650 (1997); V. Kondratovich and J. B. Delos, Phys. Rev. A 57, 4604 (1998). 